\title{
Formation and reactions of alkylzinc reagents in room temperature ionic liquids.
}

\author{
Man Chun Law, ${ }^{a b}$ Kwok-Yin Wong ${ }^{b}$ and Tak Hang Chan ${ }^{\star a b}$ \\ ${ }^{a}$ Department of Chemistry, McGill University, Montreal, Quebec Canada H3A 2K.; \\ ${ }^{b}$ Department of Applied Biology and Chemical Technology, The Hong Kong \\ Polytechnic University, Hung Hom, Hong Kong SAR, China. \\ E-mail: tak-hang.chan@mcgill.ca
}

Table of content:

Figure 1. Cyclic voltammograms of $[\mathrm{bpy}]\left[\mathrm{BF}_{4}\right]$ containing various concentrations of $[\mathrm{bpy}][\mathrm{Br}] \quad$ p.S2

Figure 2. Calibration curve of bromide concentration in $[\mathrm{bpy}]\left[\mathrm{BF}_{4}\right]$ against current $\quad$ p.S3

Figure 3. Colorless solution of [bpy][Br] dissolved in pure $[\mathrm{bpy}]\left[\mathrm{BF}_{4}\right] \quad$ p.S4

Figure 4. Blue color solution of metallic zinc in $[\mathrm{bpy}]\left[\mathrm{BF}_{4}\right]$ (bromide containing) p.S4

Figure 5. Green color solution of ethylzinc species in IL p.S5

Figure 6. Benzaldehyde added to the ethylzinc speces in IL p.S5

Figure 7. ${ }^{1} \mathrm{H}$ NMR spectrum of isolated compound 3a $\quad$ p.S6

Figure 8. ${ }^{1} \mathrm{H}$ NMR spectrum of isolated compound $\mathbf{3 b} \quad$ p.S6

Figure 9. ${ }^{1} \mathrm{H}$ NMR spectrum of isolated compound 1-( $p$-chlorophenyl)-propanol 3c $\quad$ p.S7

Figure 10. ${ }^{1} \mathrm{H}$ NMR spectrum of isolated compound 1-(p-cyanophenyl)-propanol 3d $\quad$ p.S7

Figure 11. ${ }^{1} \mathrm{H}$ NMR spectrum of isolated compound 1-( $p$-methoxyphenyl)-propanol 3e $\quad$ p.S8

Figure 12. ${ }^{1} \mathrm{H}$ NMR spectrum of isolated compound 1-(p-bromophenyl)-propanol 3f $\quad$ p.S8

Figure 13. ${ }^{1} \mathrm{H}$ NMR spectrum of isolated compound 1-(2'-pyridinyl)-propanol 3i $\quad$ p.S9

Figure 14. ${ }^{1} \mathrm{H}$ NMR spectrum of isolated compound 1-(2,6-dichlorophenyl)-propanol 3g $\quad$ p.S9

Figure 15. ${ }^{1} \mathrm{H}$ NMR spectrum of isolated compound 1-(phenyl)-pent-1-en-3-ol 3h p.S10 


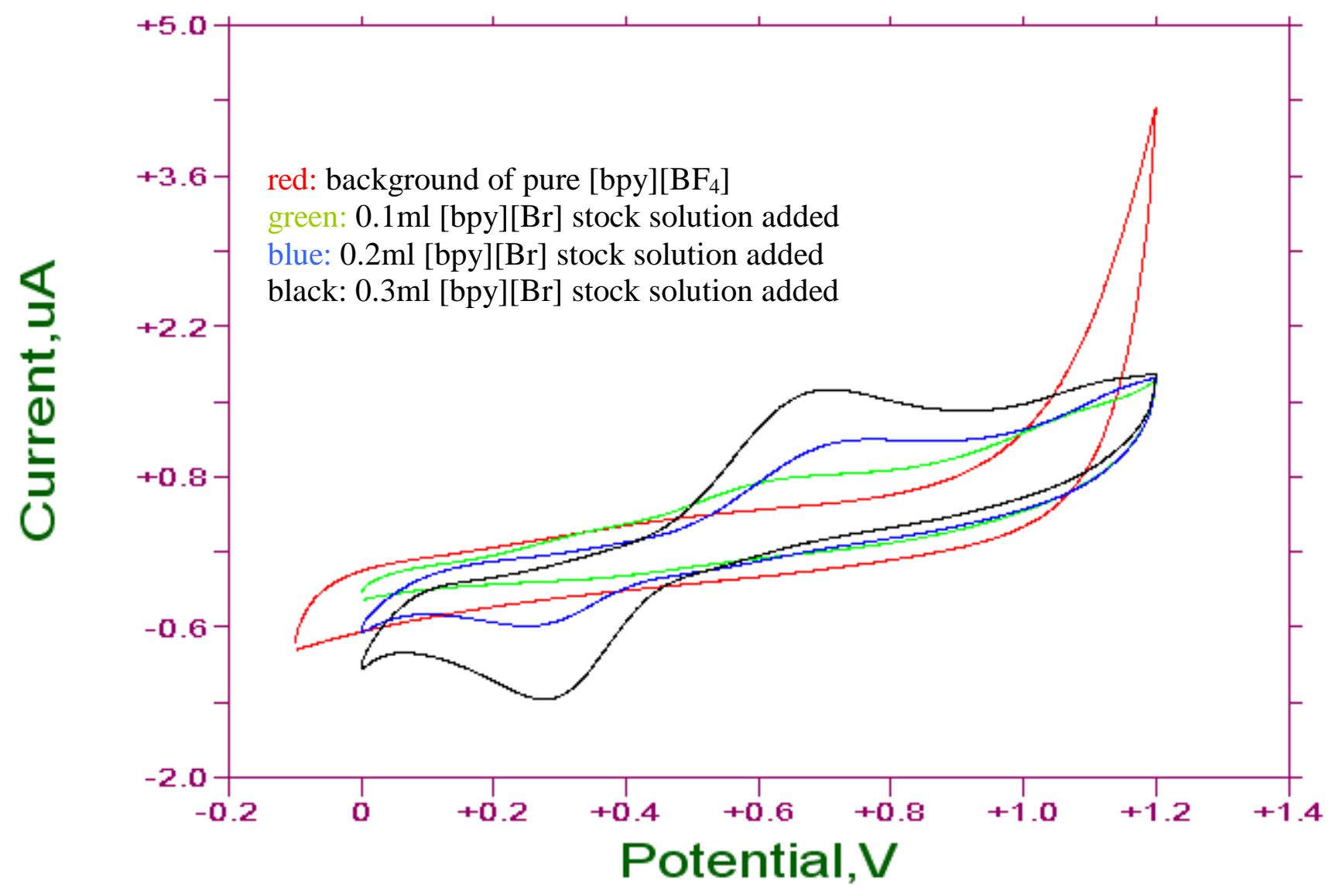

Figure 1. Cyclic voltammograms of $[\mathrm{bpy}]\left[\mathrm{BF}_{4}\right]$ containing various concentrations of $[\mathrm{bpy}][\mathrm{Br}]$. 


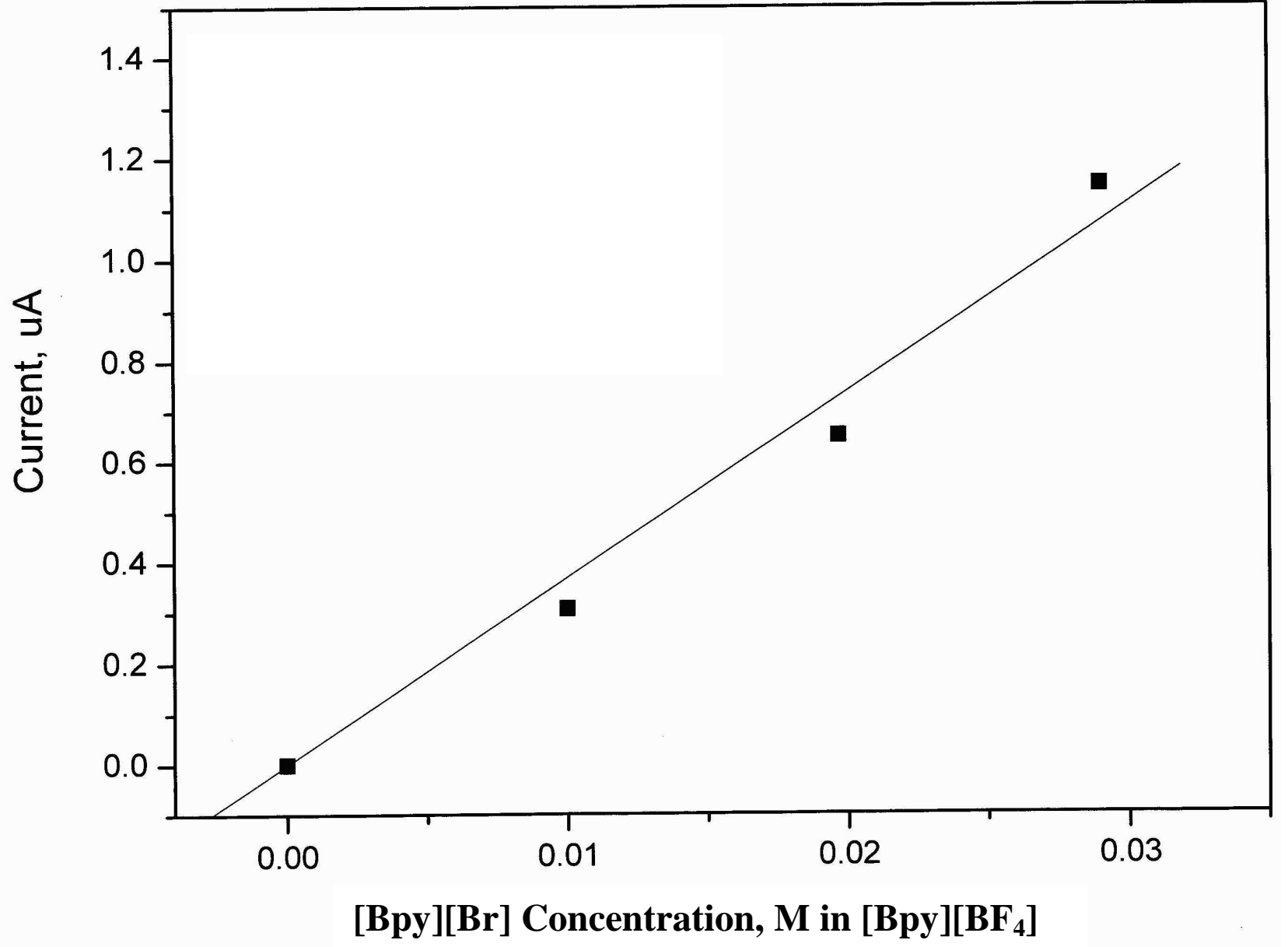

Figure 2. Calibration curve of bromide concentration in $[\mathrm{bpy}]\left[\mathrm{BF}_{4}\right]$ against current. 


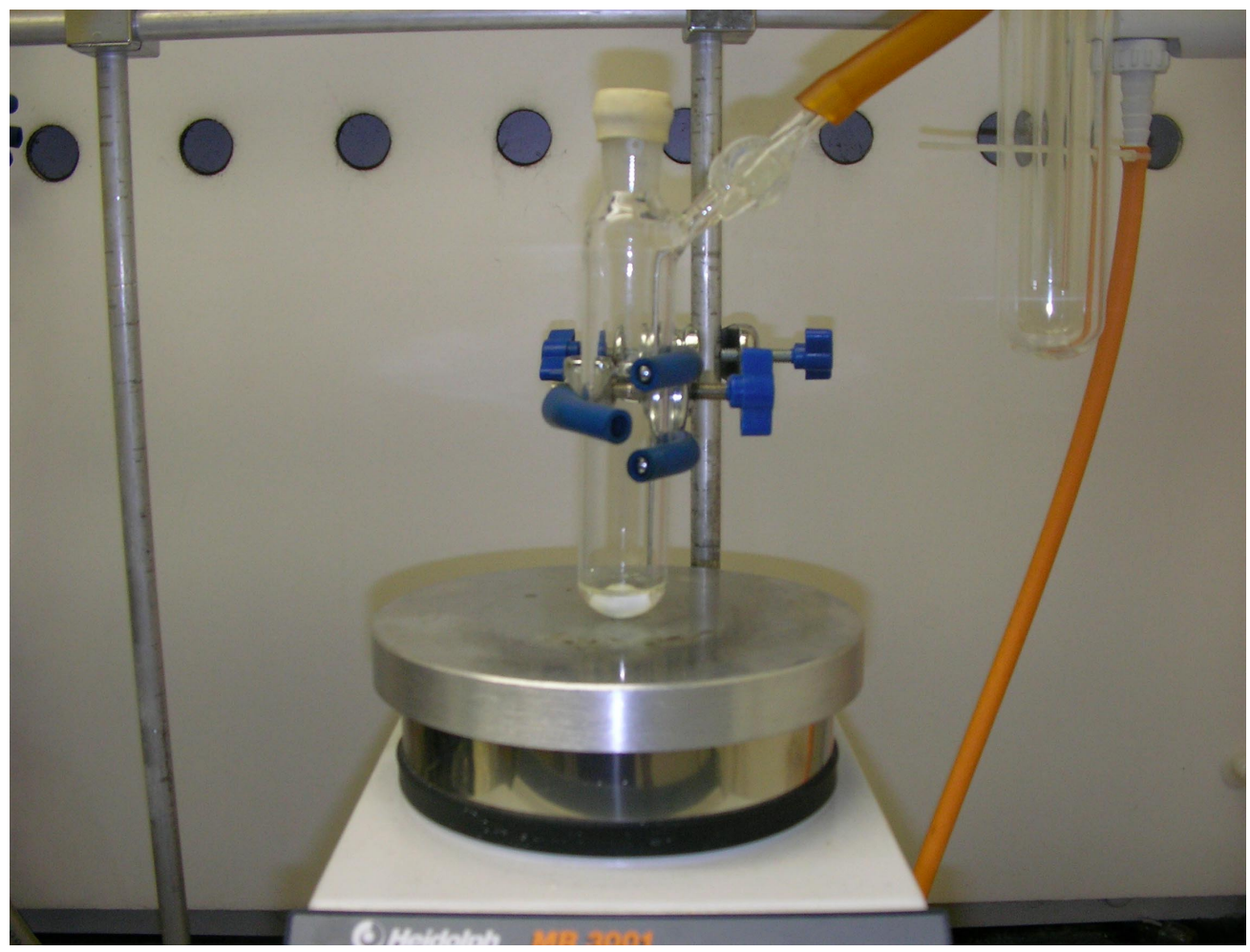

Figure 3: Colorless solution of $[\mathrm{bpy}][\mathrm{Br}]$ dissolved in pure $[\mathrm{bpy}]\left[\mathrm{BF}_{4}\right]$

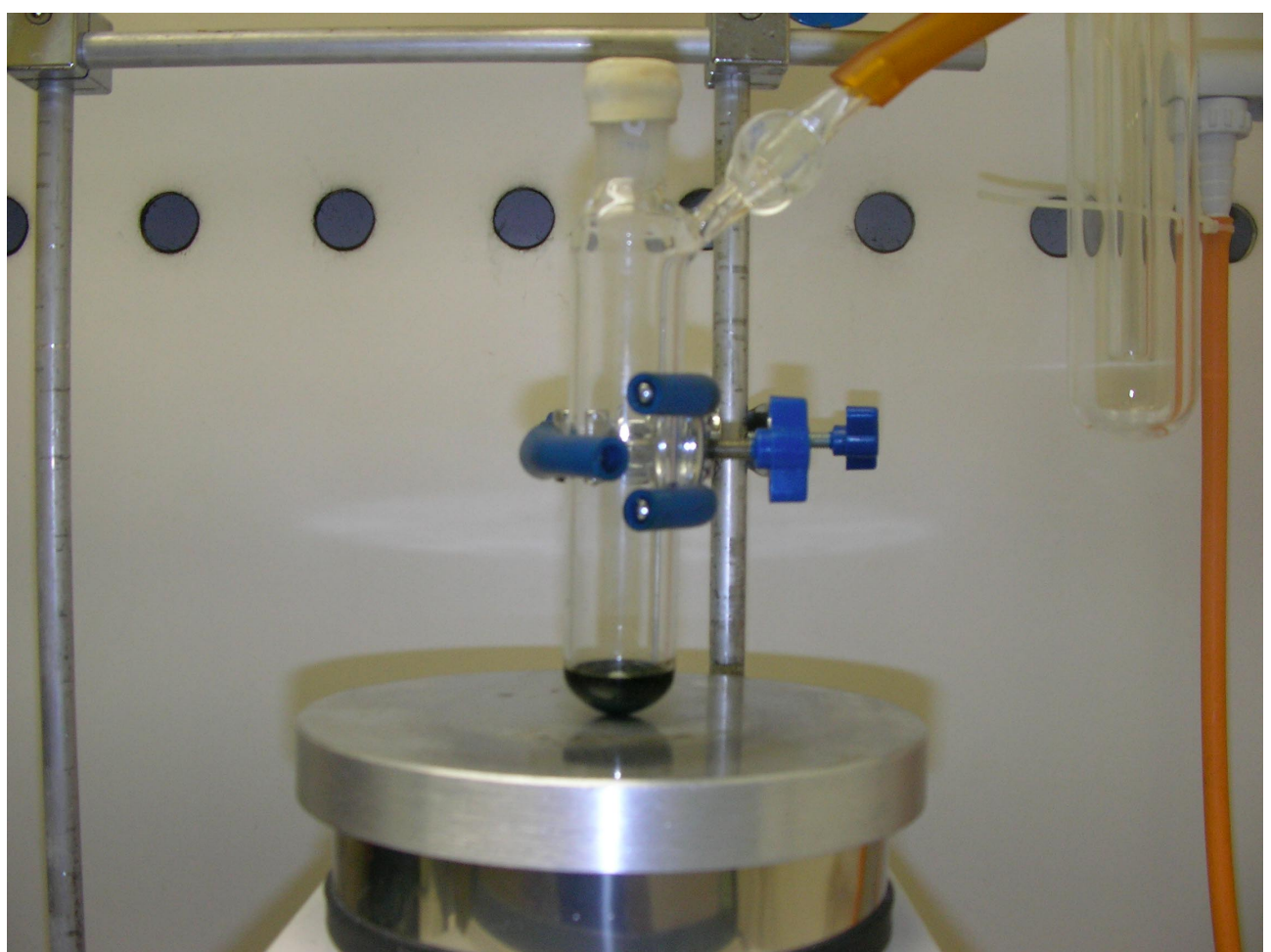

Figure 4: Blue color solution of metallic zinc in $[\mathrm{bpy}]\left[\mathrm{BF}_{4}\right]$ (bromide containing). 


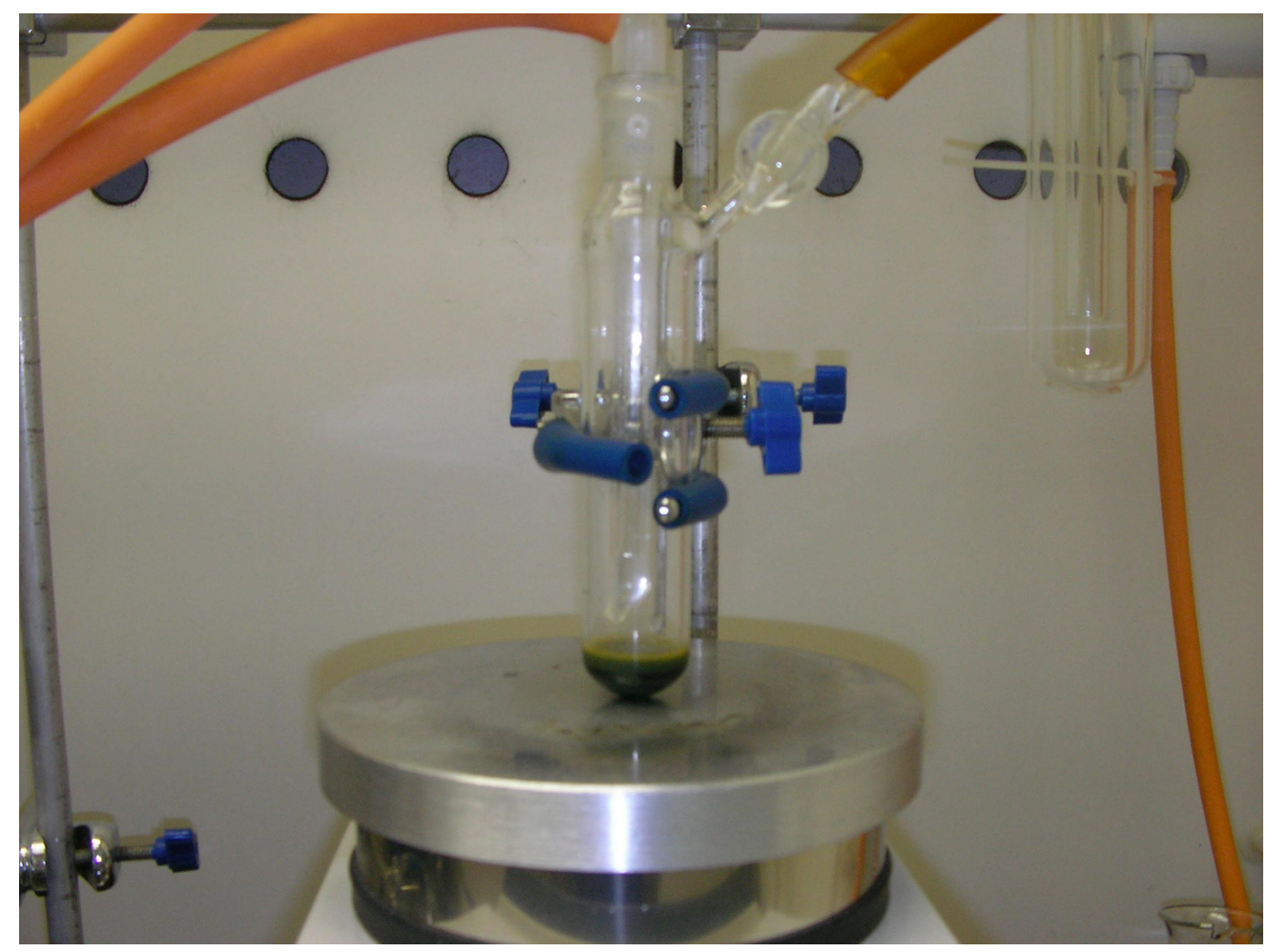

Figure 5: Green color solution of ethylzinc species in IL

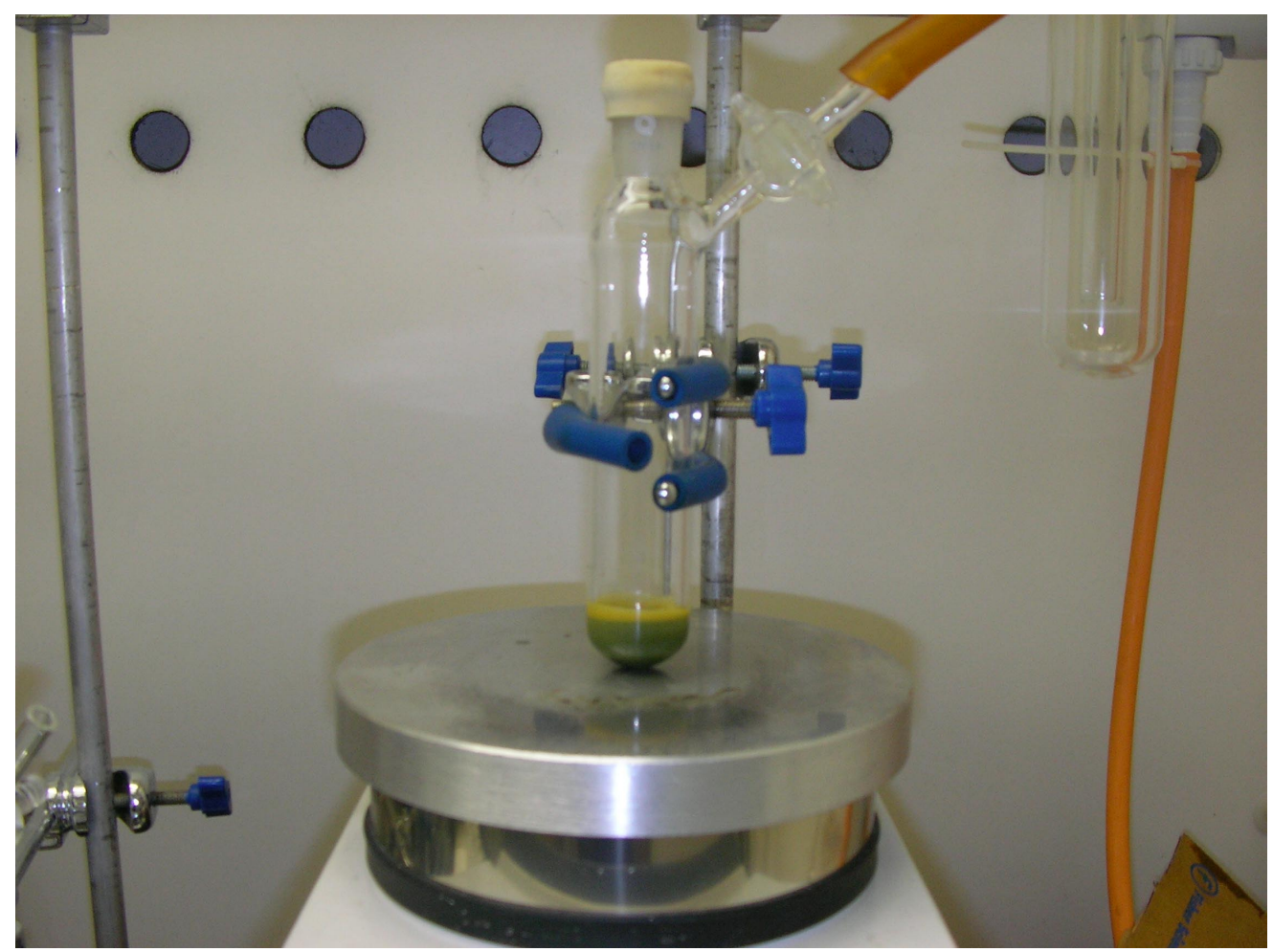

Figure 6: Benzaldehyde added to the ethylzinc speces in IL 


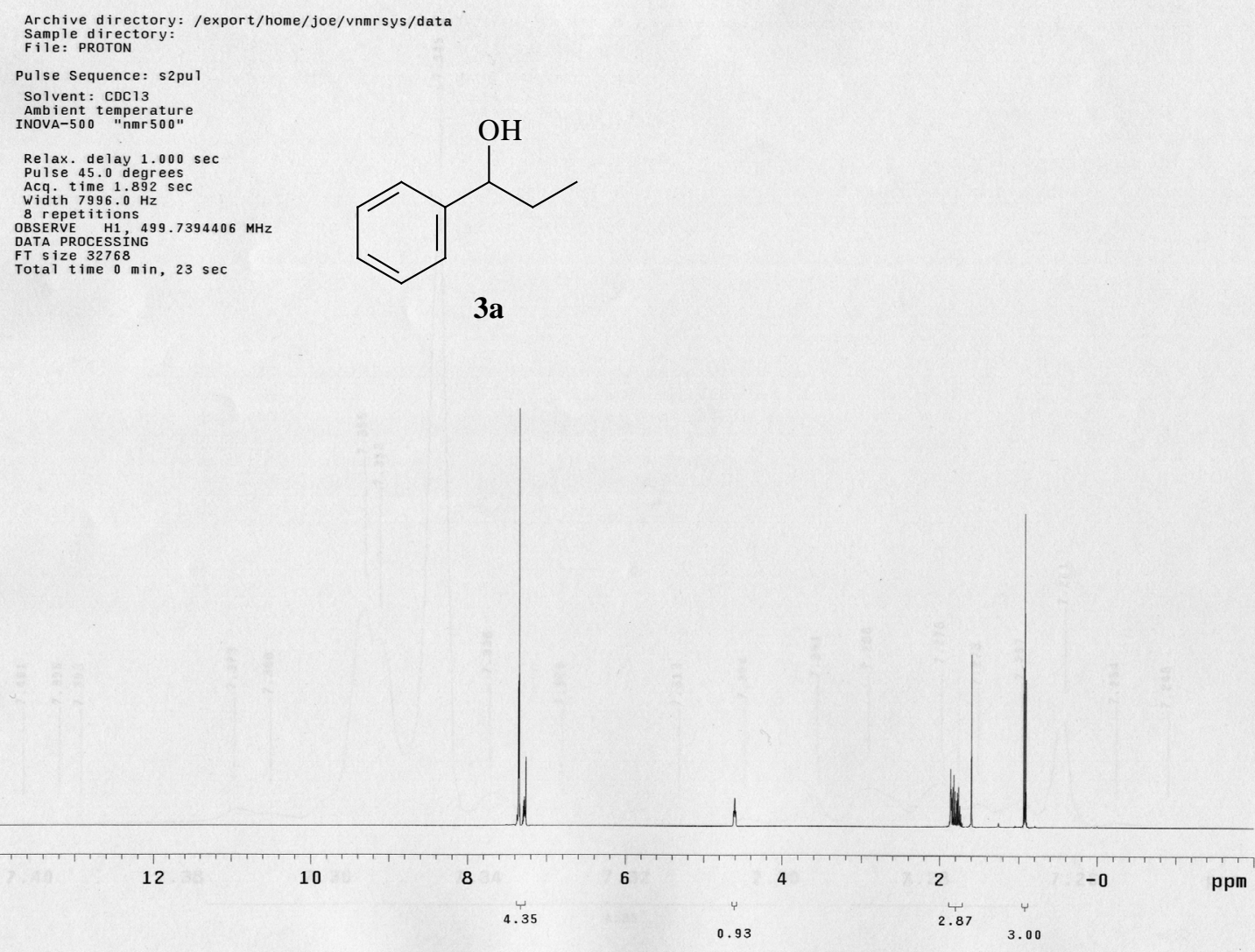

Figure 7: ${ }^{1} \mathrm{H}$ NMR spectrum of isolated compound 3a

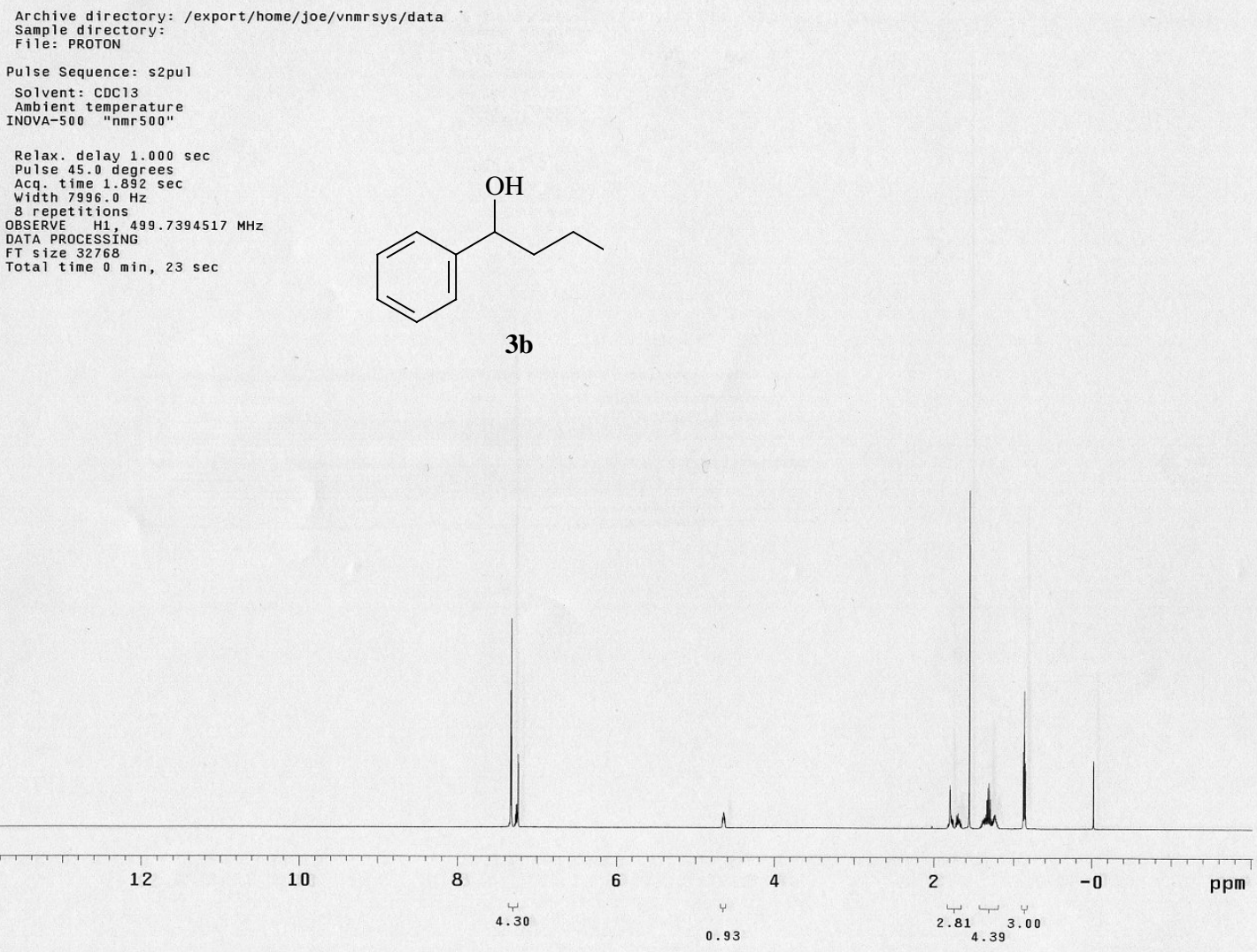

Figure 8: ${ }^{1} \mathrm{H}$ NMR spectrum of isolated compound $\mathbf{3 b}$ 


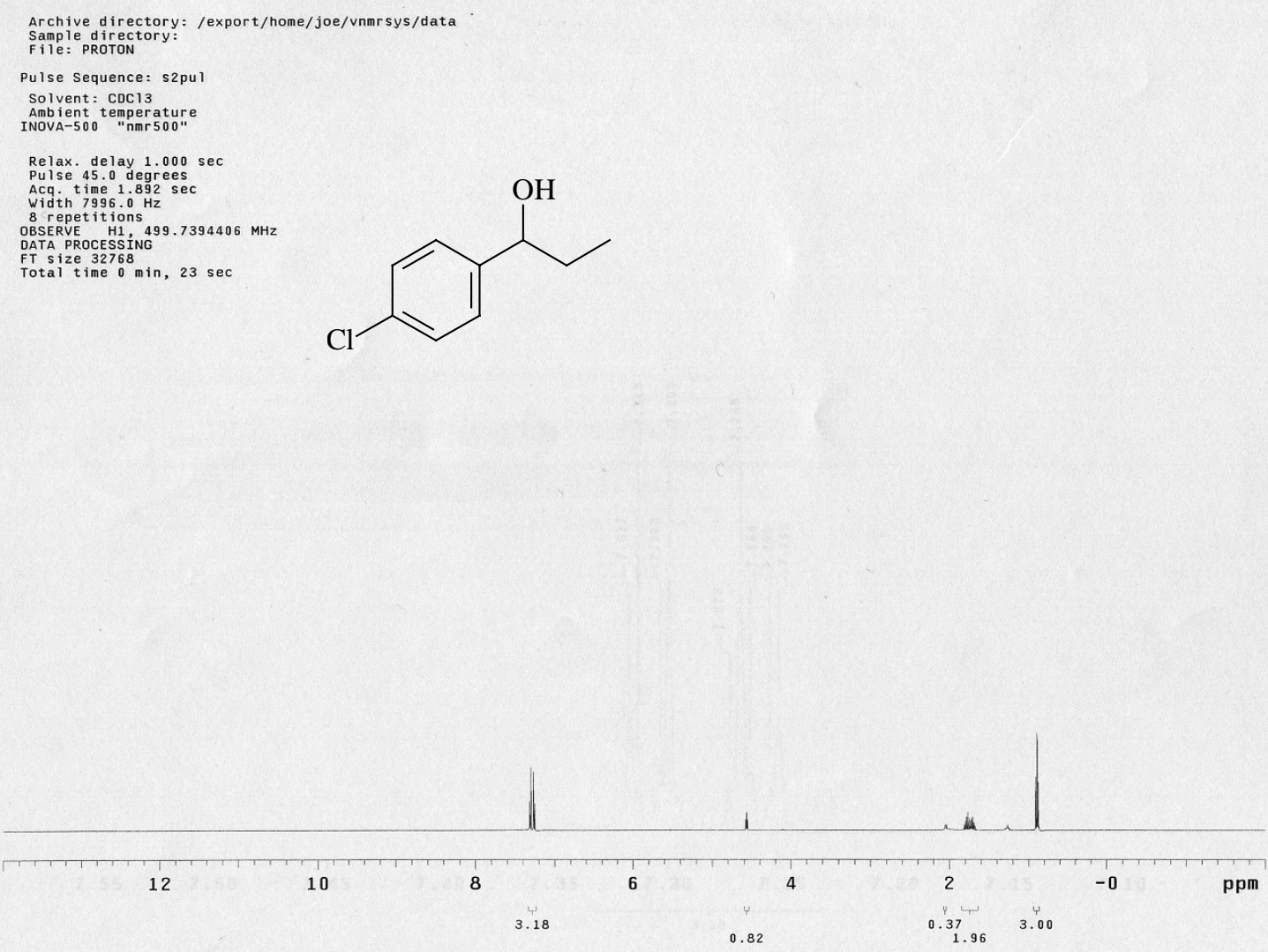

Figure 9: ${ }^{1} \mathrm{H}$ NMR spectrum of isolated compound 1-( $p$-chlorophenyl)-propanol 3c Archive directory: /export/home/joe/vnmrsys/data
Sample directory: Sample directory:
File: PROTON

Pulse Sequence: s2pur

Solvent: $\operatorname{CDCl} 13$
Ambient temperature

Relax. delay 1.000 sec

Relax. delay 1.000 sec
Pulse 45.0 degrees

Acq. time 1.892 se

8 repetitions

OBSERVE H1, $499.7394522 \mathrm{MHZ}$

DATA RROCESSING
Fize 32768 .
Total time 0 min, $23 \mathrm{sec}$<smiles>CCC(O)c1ccc(C#N)cc1</smiles>

Thathes
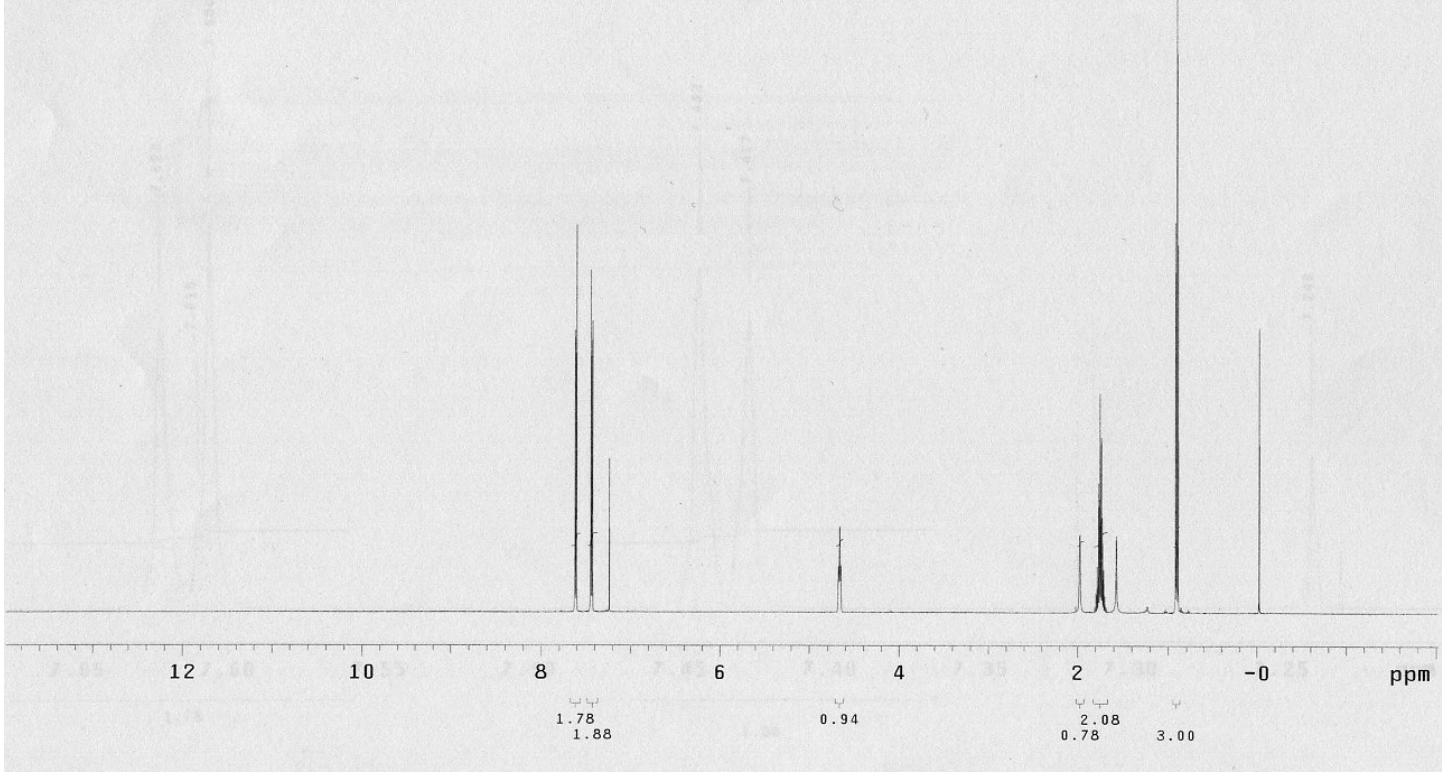

Figure 10: ${ }^{1} \mathrm{H}$ NMR spectrum of isolated compound 1-(p-cyanophenyl)-propanol 3d 


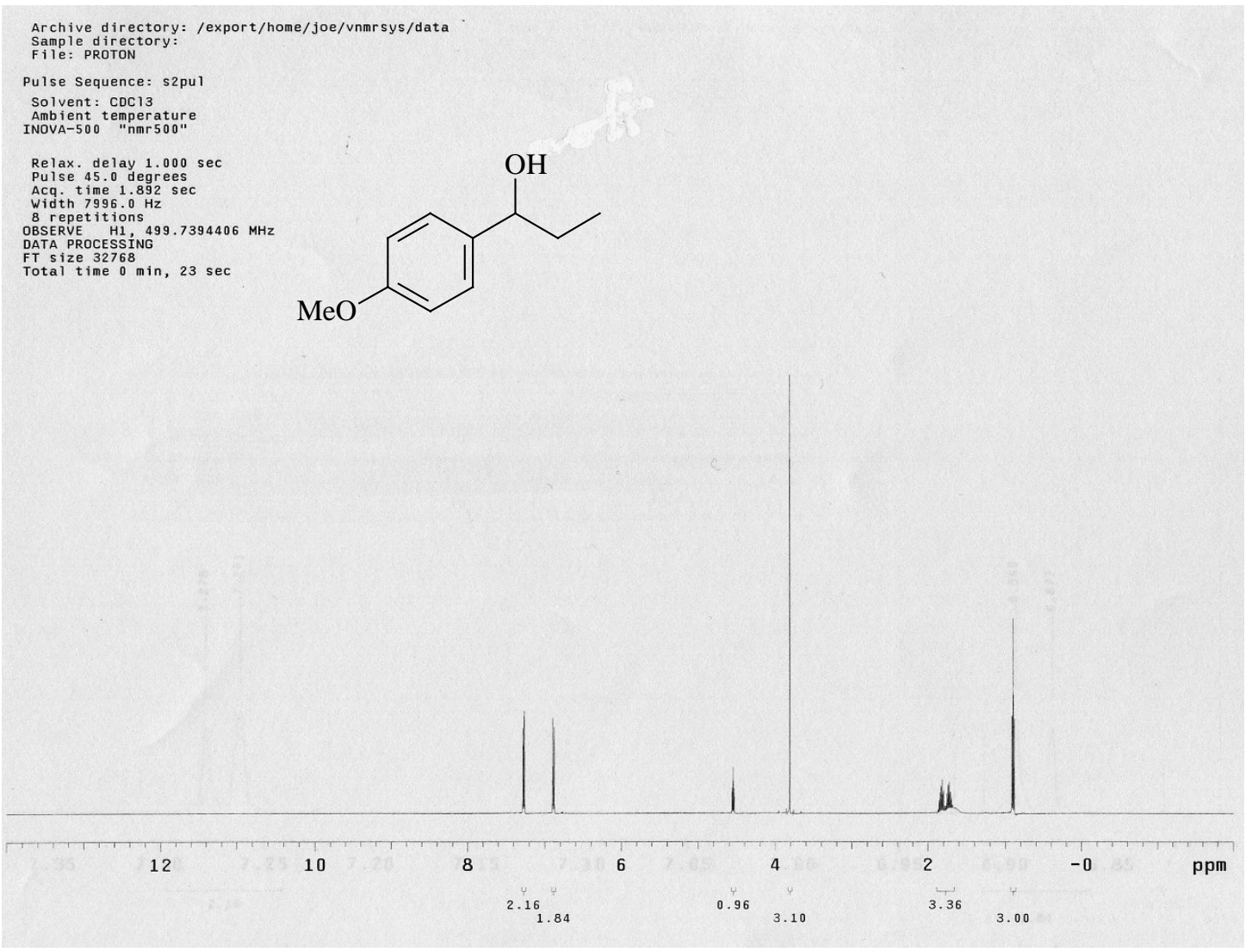

Figure 11: ${ }^{1} \mathrm{H}$ NMR spectrum of isolated compound 1-(p-methoxyphenyl)-propanol 3e Archive directory: /export/home/joe/vnmrsys/data Sample directory:
File: PROTON

Pulse Sequence: s2pul

Solvent: $\operatorname{CDCl}_{3}$

Amb ient temperature
INOVA 500 "nmr500"

Relax. delay $1.000 \mathrm{sec}$
Pulse 45.0 degrees

Pulse 45.0 degrees
Acr. time $1.892 \mathrm{sec}$
Width $7996.0 \mathrm{~Hz}$

8 repetitions

DATA PROCESSING

FT size 32768
Total time $0 \mathrm{~min}, 23 \mathrm{sec}$<smiles>CCC(O)c1ccc(Br)cc1</smiles>

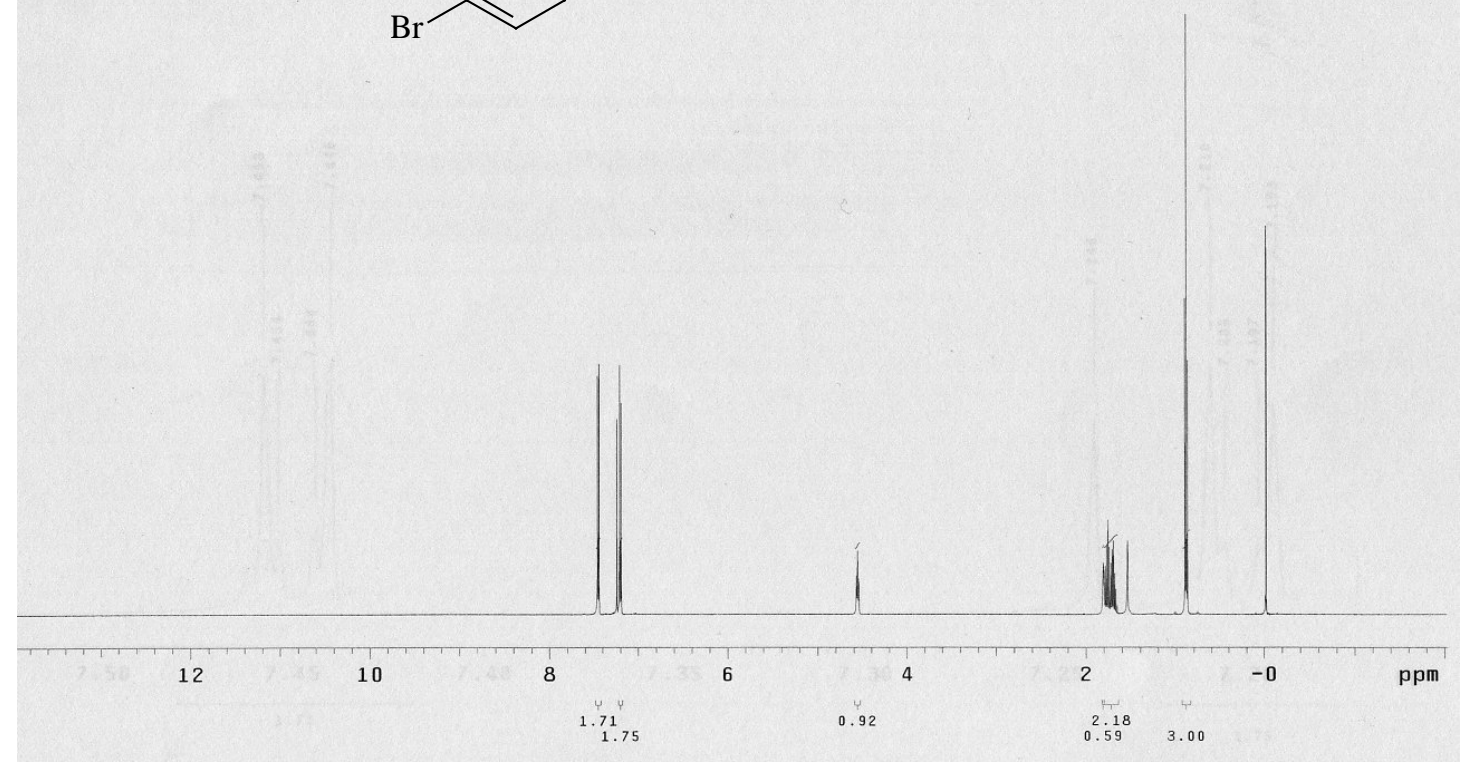

Figure 12: ${ }^{1} \mathrm{H}$ NMR spectrum of isolated compound 1-(p-bromophenyl)-propanol $\mathbf{3 f}$ 


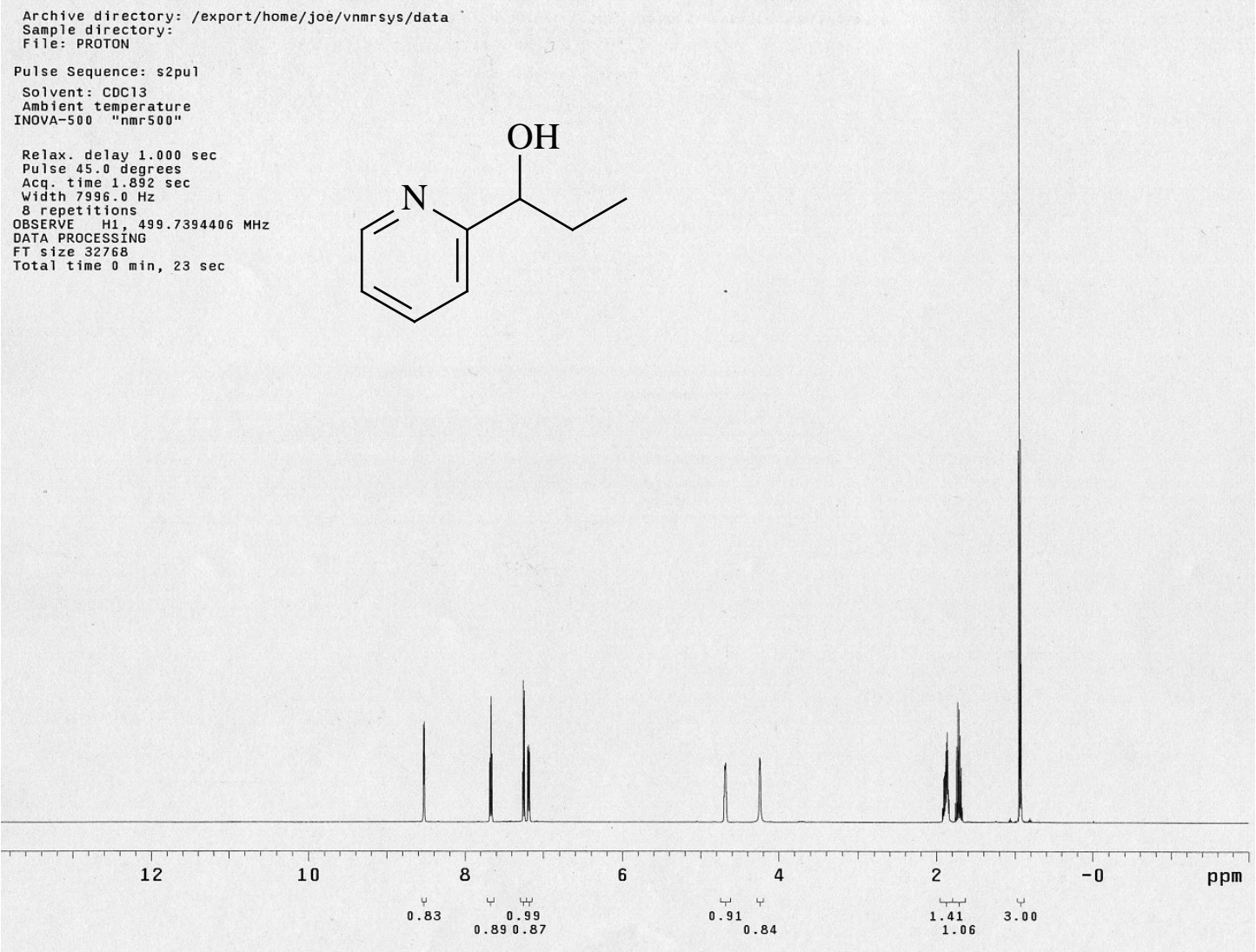

Figure 13: ${ }^{1} \mathrm{H}$ NMR spectrum of isolated compound 1-(2'-pyridinyl)-propanol 3i
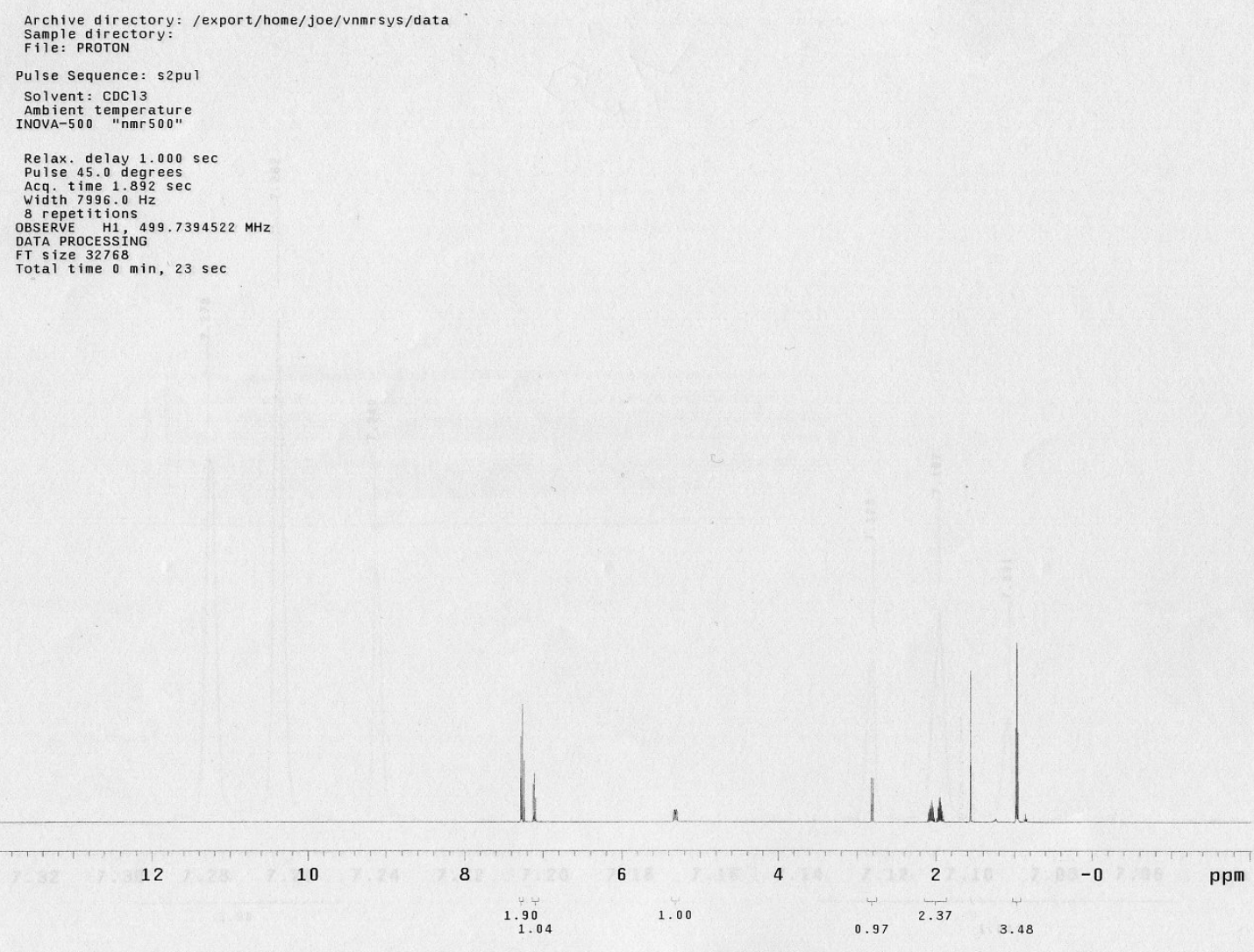

Figure 14: ${ }^{1} \mathrm{H}$ NMR spectrum of isolated compound 1-(2,6-dichlorophenyl)-propanol $\mathbf{3 g}$ 


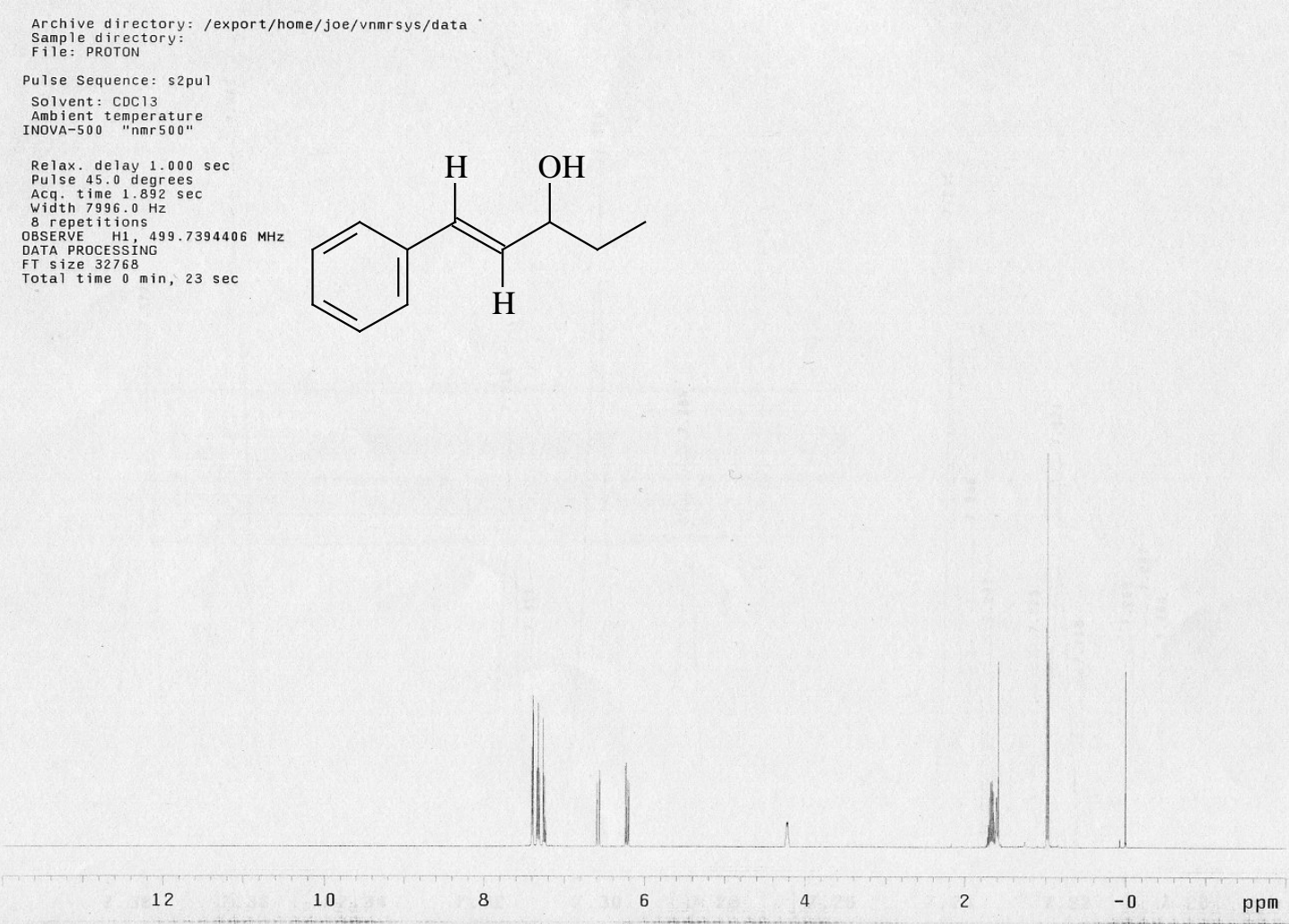

Figure 15: ${ }^{1} \mathrm{H}$ NMR spectrum of isolated compound 1-(phenyl)-pent-1-en-3-ol $\mathbf{3 h}$ 